\title{
Less than perfect divorces: dysregulated mitochondrial fission and neurodegeneration
}

\author{
Björn Oettinghaus · Maria Licci · Luca Scorrano • \\ Stephan Frank
}

Received: 19 October 2011/Revised: 6 December 2011/Accepted: 7 December 2011/Published online: 17 December 2011

(C) Springer-Verlag 2011

\begin{abstract}
Research efforts during the last decade have deciphered the basic molecular mechanisms governing mitochondrial fusion and fission. We now know that in mammalian cells mitochondrial fission is mediated by the large GTPase dynamin-related protein 1 (Drp1) acting in concert with outer mitochondrial membrane (OMM) proteins such as Fis1, Mff, and Mief1. It is also generally accepted that organelle fusion depends on the action of three large GTPases: mitofusins (Mfn1, Mfn2) mediating membrane fusion on the OMM level, and Opa1 which is essential for inner mitochondrial membrane fusion. Significantly, mutations in Drp1, Mfn2, and Opa1 have causally been linked to neurodegenerative conditions. Despite this knowledge, crucial questions such as to how fission of the inner and outer mitochondrial membranes are coordinated and how these processes are integrated into basic physiological processes such as apoptosis and autophagy remain to be answered in detail. In this review,
\end{abstract}

B. Oettinghaus and M. Licci contributed equally to this work.

B. Oettinghaus $\cdot$ M. Licci $\cdot$ L. Scorrano

Department of Cell Physiology and Metabolism,

University of Geneva Medical School, Geneva, Switzerland

B. Oettinghaus · M. Licci · S. Frank $(\bowtie)$

Division of Neuropathology, Institute of Pathology, University

Hospital Basel, Schönbeinstr. 40, 4031 Basel, Switzerland

e-mail: frankst@uhbs.ch

M. Licci

Department of Neurosurgery, University Hospital Basel,

Basel, Switzerland

L. Scorrano

Dulbecco-Telethon Institute, Venetian Institute of Molecular

Medicine, Padua, Italy we will focus on what is currently known about the mechanism of mitochondrial fission and explore the pathophysiological consequences of dysregulated organelle fission with a special focus on neurodegenerative conditions, including Alzheimer's, Huntington's and Parkinson's disease, as well as ischemic brain damage.

\section{Introduction}

Mitochondria play important roles in life and death of eukaryotic cells, providing most of the ATP required for metabolic cell processes via oxidative phosphorylation. Moreover, in addition to regulating several other metabolic pathways, such as Krebs cycle, fatty acid metabolism, gluconeogenesis, and heme-synthesis, mitochondria also control intracellular calcium signaling and apoptosis.

Although early high-resolution electron microscopy images of the organelles - which were obtained by Palade in the early 50s and which have since coined the standard depictions for ensuing text book generations-displayed static, bean-shaped, double-membrane mitochondrial units [80], observations of living cells dating back to 1914 had already revealed that mitochondria are extremely dynamic, modulating their morphology through fusion and fission processes that create highly interconnected tubular networks as well as punctiform solitary organelles [55]. These continuous changes in mitochondrial morphology, driven by antagonistic, normally balanced fusion and fission processes are controlled by a group of evolutionary-conserved large GTPases that share structural homology with prototypical dynamins [96]. Dynamins, ranging in size between 70 and $100 \mathrm{kDa}$, are mechanochemical enzymes thatthrough GTP hydrolysis-driven assembly into multimeric 
spirals around the collars of invaginating vesicles-are mainly involved in the scission of nascent endo- and exocytic vesicles $[53,63,96]$.

\section{Mitochondrial fusion}

In mammals, the dynamin-like GTPases mitofusin 1 and 2 (Mfn1, Mfn2) regulate OMM fusion, whereas the integral IMM protein OPA1-in addition to mediating mitochondrial fusion in concert with Mfn1-mediates apoptotic processes by regulating mitochondrial cristae maintenance and cytochrome $c$ release. Whereas in the most experimental paradigms a fused mitochondrial phenotype seems to protect cells from programmed cell death, forced activation of organelle fission favors apoptosis by facilitating the release of cytochrome $c$ from the mitochondria with the subsequent activation of effector caspases.

Both mitofusins, Mfn1 and Mfn2, are integrated into the OMM via a bipartite transmembrane domain, with their $\mathrm{C}$ - and $\mathrm{N}$-termini including the GTPase domain facing the cytosol. Via formation of homo- as well as heterodimers, Mfns are thought to mediate the initial step of organelle tethering. Whereas Mfn1 exhibits higher GTPase and organelle fusion activity, Mfn2 features higher substrate affinity toward GTP [44]. In addition, Mfn2, but not Mfn1, is expressed on endoplasmic reticulum (ER) membranes and is thus thought to be also important for ER-mitochondria interactions including calcium homeostasis [25].

Interestingly, OPA1 germline mutations cause autosomal-dominant optic atrophy [1,27], whereas Mfn2 germline mutations are causally linked to an axonal variant of Charcot-Marie-Tooth disease (CMT 2A) [122]. Of note, genetic ablation of Mfn2 in spinal cord motor neurons leads to their degeneration resulting in subsequent hindlimb paralysis [28]. In addition, neurodegeneration is also prominent in mice with cerebellar or Purkinje neuron-specific Mfn2 deletions [16]. Last but not least, mice in which Mfn1 and Mfn2 have been co-deleted specifically in skeletal muscle develop a mitochondrial myopathy-like phenotype which recapitulates the typical histological findings of SDH-hyperreactive/COX-negative muscle fibres and subsarcolemmal mitochondrial accumulations, accompanied by a pronounced depletion of mtDNA [17].

\section{Mitochondrial fission}

Dynamin-related protein 1 (Drp1) displays a high degree of evolutionary conservation and is the only known large GTPase actively mediating mitochondrial fission. In humans, Drp1 is highly expressed in tissues with particular energy demands, such as brain and muscle, whereas moderate-to-low expression levels are found in liver, lung, placenta, kidney, and pancreas [96].

The molecular structure of Drp1 largely corresponds to that of other proteins of the dynamin superfamily, except for the lack of the $\mathrm{C}$-terminal proline-rich domain present in most members of the superfamily and a lacking pleckstrin homology domain $(\mathrm{PH})$ which mediates proteinmembrane interactions in dynamins [96] (Fig. 1). The recently resolved crystal structure of dynamin shows three protein-protein interfaces that allow the formation of multimers. Detailed analysis of interface 2 reveals a conserved amino-acid pattern that is unique to Drp1 and probably responsible for its dimerization [33]. On the cellular level, almost the entire Drp1 dimer pool is cytosolic with only about $3 \%$ of the total protein content residing at the mitochondrial surface. In order to induce organelle fission, cytosolic Drp1 requires activation and subsequent translocation to the mitochondrial outer membrane. Here, Drp1 dimers form ring-like multimeric structures on prospective OMM fission sites through a self-assembling process [43], followed by GTP hydrolysis-driven conformational changes that-via ring constriction-lead to membrane severing and subsequent organelle division [53, 63] (Fig. 1c). Accordingly, integration of mutated Drp1 molecules (e.g., of the K38A mutation with incapacitated GTP hydrolysis) into the ringlike Drp1 multimers inhibits their constriction through dominant-negative effects, resulting in blocked organelle fission [96] (Fig. 2). Recently, cryo-EM studies of Dnm1, the yeast orthologues of Drp1, provided new insights into its contractile mechanochemical properties. The magnitude of Drp1 constriction was found to be substantially larger than that of dynamin. A derived model postulates that Drp1 forms multimeric constrictive rings that engage in only limited interaction with the underlying lipid bilayer leaving a 3- to 4-nm pitch, indicating that Drp1 membrane interactions may involve adaptor proteins to bridge that gap [63].

The OMM-bound mitochondrial fission factor (Mff) has recently been identified as such an adaptor protein for Drp1 [79] (Fig. 1a). In addition, OMM-bound mitochondrial elongation factor 1 (Mief1) facilitates mitochondrial Drp1 translocation [121]. Curiously, by blocking GTP binding at the GTPase domain of Drp1, Mief1 inhibits the conformational change of Drp1 multimers required for membrane fission [121] (Fig. 1d). According to a recently proposed model, expression of mammalian Fis1-another integral OMM protein [48] - is able to partially reverse an elongated mitochondrial phenotype by sequestering Mief1 via unblocking Drp1 GTP hydrolysis, culminating in organelle fission [121] (Fig. 1e). 


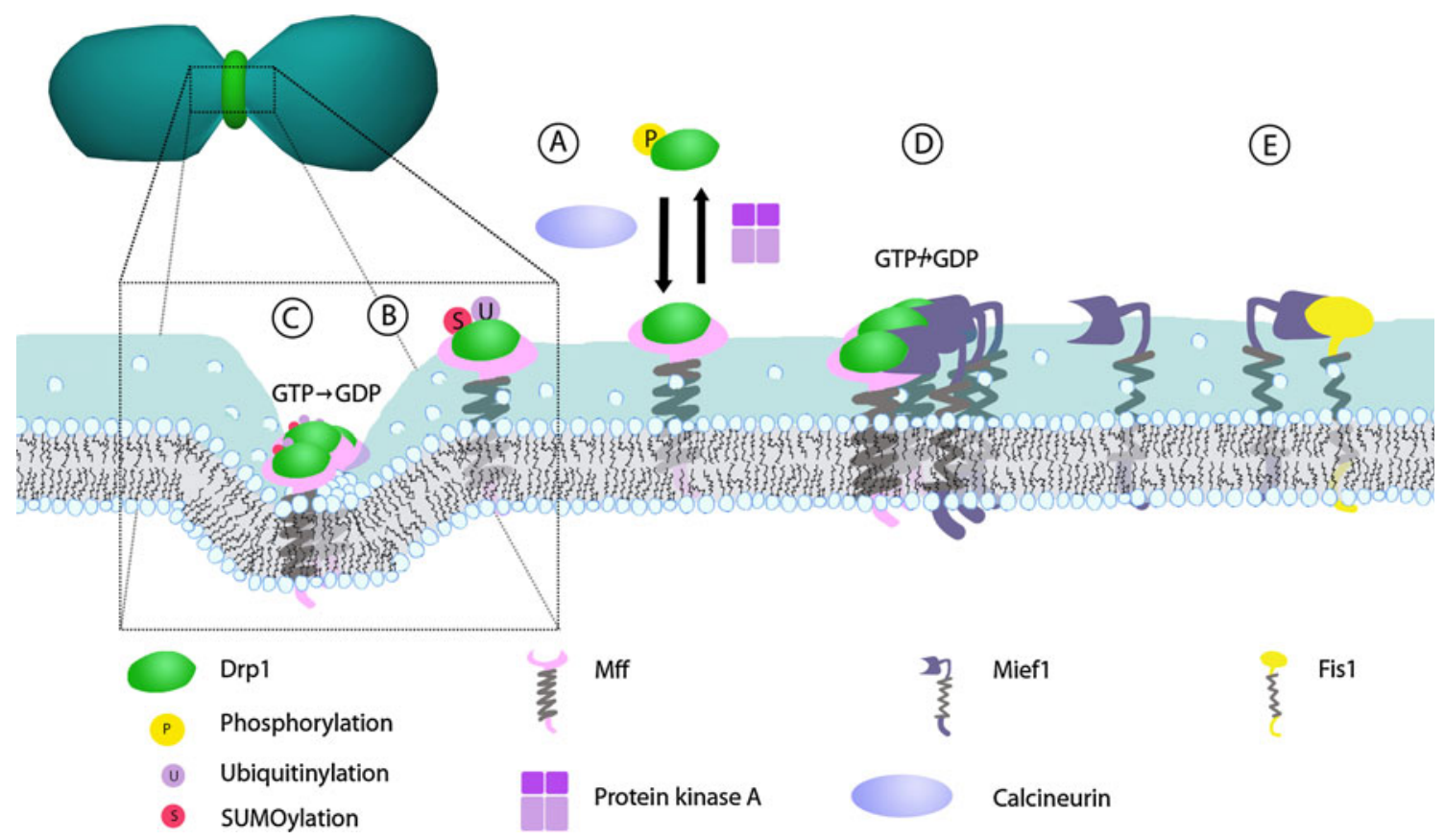

Fig. 1 Molecular regulation of Drp1-mediated mitochondrial fission. a Activation of Drp1 by, e.g., calcineurin-mediated dephosphorylation targets the protein to mitochondria where it is thought to bind the adaptor protein Mff. b Additional posttranslational Drp1 modifications such as ubiquitination and sumoylation stabilize Drp1 on the OMM. c Following oligomerization, Drp1 assembles into constrictive ring-like multimers that, upon GTP hydrolysis, mediate membrane constriction. d Binding of Mief1 to Drp1 on the OMM inhibits GTP hydrolysis and subsequent membrane constriction. e The OMM protein Fis1 likely exerts its pro-fission activity by sequestering Mief1, thereby inhibiting Drp1-Mief1 interactions

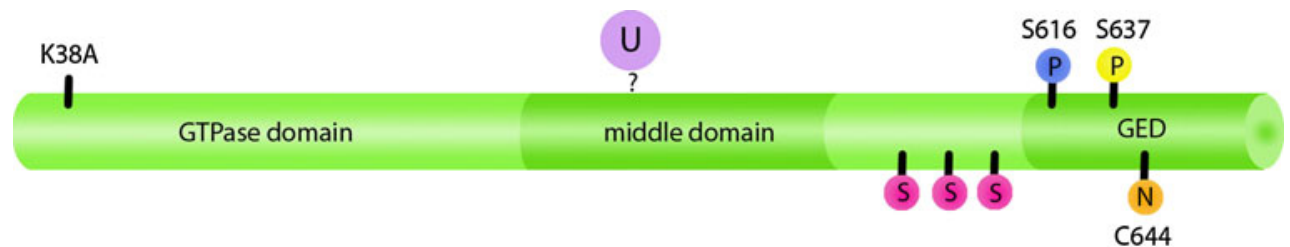

\section{S SUMOylation P Drp1 inactivating phosphorylation \\ N S-Nitrosylation \\ P Drp1 activating phosphorylation}

Ubiquitinylation

Fig. 2 Drp1 structure and posttranslational modifications. Drp1 is composed of a N-terminal GTPase domain, a middle domain and a C-terminal GTPase effector domain (GED), the latter-through impacting GTP hydrolysis-influencing Drp1 oligomerization. The K38A mutation alters catalytic core residues within the GTPase domain; however, K38A mutant Drp1 can still oligomerize with wildtype Drp1, thus acting as a dominant-negative mutation. The E3ligases March5 and parkin can ubiquitinate Drp1 at currently unknown sites. March 5-mediated ubiquitination stabilizes the mitochondrial Drp1 pool while parkin-mediated ubiquitination targets Drp1 for proteasomal degradation. Phosphorylation of Drp1 can exert

Interestingly, a very recent report also indicates that interorganellar contact sites between mitochondria and adjacent endoplasmic reticulum membranes are important for the mitochondrial division process by constricting mitochondria at prospective fission sites, prior to the recruitment of any of the fission-mediating proteins discussed above [36]. either activating or inactivating effects. Drp1 phosphorylation at S616 by the CDK1/CyclinB complex triggers Drp1-mediated mitochondrial fission during mitosis. In contrast, PKA-mediated phosphorylation at S637 inhibits mitochondrial Drp1 translocation; this effect can be antagonized by the phosphatase calcineurin. Sumoylation of Drp1 can occur at multiple sites between the middle and GED domains, and is mediated by various ubiquitin ligases such as Sumo1, Ubc9, and MAPL. Sumoylation of Drp1 stabilizes its association with mitochondria. Finally, mitochondrial fission in the context of Alzheimer's disease involves $A \beta$-mediated S-nitrosylation of Drp1 at position C644

\section{Posttranslational Drp1 modifications}

Changes in mitochondrial shape are tightly coupled to essential organellar and cellular functions, as exemplified during mitosis, when the balance between fusion and fission temporarily shifts toward fission to enable equal mitochondrial distribution to daughter cells [100]. In order 
to allow for rapid adaptation to continuously changing cellular needs, extensive post-translational modifications such as phosphorylation, sumoylation and ubiquitination are in place, which, by regulating the activity of mitochondrial morphogenic proteins, directly impinge on organelle morphology (Fig. 2). When activated by increased cAMP levels, protein kinase A (PKA) phosphorylates Drp1 at residue Ser637, thereby inhibiting its GTPase activity as well as intermolecular Drp1 interactions resulting in elongated mitochondria $[14,22]$. This mechanism is being exploited during starvation where mitochondrial elongation resulting from PKA-mediated Drp1 phosphorylation can protect starving cells from autophagy [38]. PKA-mediated Drp1 phosphorylation at Ser 637 is counteracted by calcineurin, whose activation leads to organelle fragmentation by boosting Drp1 translocation to mitochondria $[14,112]$. As calcineurin activation occurs in response to increased cytosolic calcium levels, this mechanism could be activated in $\mathrm{Ca}^{2+}$ mediated neuronal injury, e.g., during ischemia or excitotoxicity. Of note, increased calcineurin activity and subsequent mitochondria fission were recently shown to participate in neuronal toxicity in a Huntington disease mouse model [21].

During mitosis, phosphorylation at Drp1 position S616 also activates Drp1, triggering mitochondrial fission to enable even mitochondrial distribution to cellular progeny. This phosphorylation event is mediated by the Cdk1/ CyclinB complex under the control of the small Ras-like GTPase RALA and its effector RALBP1 [51, 100]. Although cell cycle-coupled events are obviously less significant in postmitotic cells, increased levels of Drp1 S616 phosphorylation were recently found in Alzheimer's disease-affected brains [113]. In postmitotic neurons, the S616 site can alternatively be phosphorylated by protein kinase $\mathrm{C} \delta(\mathrm{PKC} \delta)$ in vitro as well as in vivo, as demonstrated in a rat model where hypertensive encephalopathy was shown to be associated with mitochondrial fission, accompanied by increased $\operatorname{Drp} 1 / \mathrm{PKC} \delta$ interactions on mitochondria [86].

In addition to the various phosphorylating modifications discussed above, Drp1 is subject to ubiquitination. To date, MARCH5 as well as parkin have been shown to act as E3 ligases with substrate specificity to Drp1. However, MARCH5-mediated ubiquitination stabilizes Drp1 on mitochondria [50, 83], while parkin-mediated ubiquitination promotes the proteasomal degradation of Drp1 [111].

Sumoylation, a related posttranslational Drp1 modification, is mediated by the small ubiquitin modifier (Sumo) which stabilizes Drp1 levels, thereby supporting mitochondrial fragmentation. Sumoylation of Drp1 is performed by Sumo1, Ubc9 and MAPL [9, 32, 41, 115, 123], while desumoylation is mediated by SenP5 [123, 124]. In the future, it will undoubtedly be interesting to test the question, in how far the impaired turnover of specific mitochondrial morphogenesis proteins is pathogenically important in the context of neurodegeneration, and whether modulating their expression levels may potentially ameliorate the clinical course of these usually relentlessly progressing diseases.

\section{Drp1 in neuronal morphogenesis, homeostasis, and neurodegeneration}

It is a well-known fact that most neurodegenerative diseases occur sporadically, with age being the most significant risk factor. Remarkably, more than one-third out of 106 genes that have so far been linked to neurodegenerative diseases, are key regulators of mitochondrial function [92]. Mutations in two of the mitochondria shaping proteins have been causally linked to familial neurodegenerative diseases. Whereas autosomal-dominant Mfn2 mutations pathogenically underlie Charcot-MarieTooth Disease type 2A (CMT2A) [122], a peripheral axonal neuropathy, Opa1 mutations lead to autosomaldominant optic atrophy (ADOA) [1, 27]. In addition, the mitochondrial fission protein Drp1 can sporadically be affected by mutations in humans, as exemplified by the report of a lethal syndromic birth defect attributable to a heterozygous Drp1 mutation [117]. In this case, a newborn presented clinically with truncal hypotonia and insufficient to absent optic and motor reflexes accompanied by persistent lactic acidemia; the infant died at day 37 after birth. Workup of that case revealed marked microcephaly, abnormal brain development with abnormal gyral patterns as well as optic atrophy. This severe developmental phenotype hinted-for the first time-at a crucial role of Drp1 for brain development. Given the early embryonic lethality of mice genetically ablated for the mitochondrial fusion proteins Mfn1, Mfn2 [15] as well as of constitutive Drp1 knockout mice (see below), it seems plausible that Drp1 mutations may cause early embryonic mortality in humans as well, with a high chance of going undetected in the clinical practice. The importance of Drp1 for the neuronal development has recently been confirmed beyond doubt by two murine models of genetic Drp1 ablation, each resulting in early embryonic lethality (e11.5). Drp1-ablated embryos display pronounced defects in the organ development affecting liver, heart and the neural tube, and lack the trophoblast giant cell layer [45, 108]. Both groups were further able to generate neuronal-specific Drp1 knockout mice using different approaches. In both models, murine embryos seem to develop normally, but die at postnatal day 0. Employing the engrailed1 (EN1) promoter, Wakabayashi and coworkers [108] ablated Drp1 specifically in the midbrain and the cerebellum, which appeared smaller, 

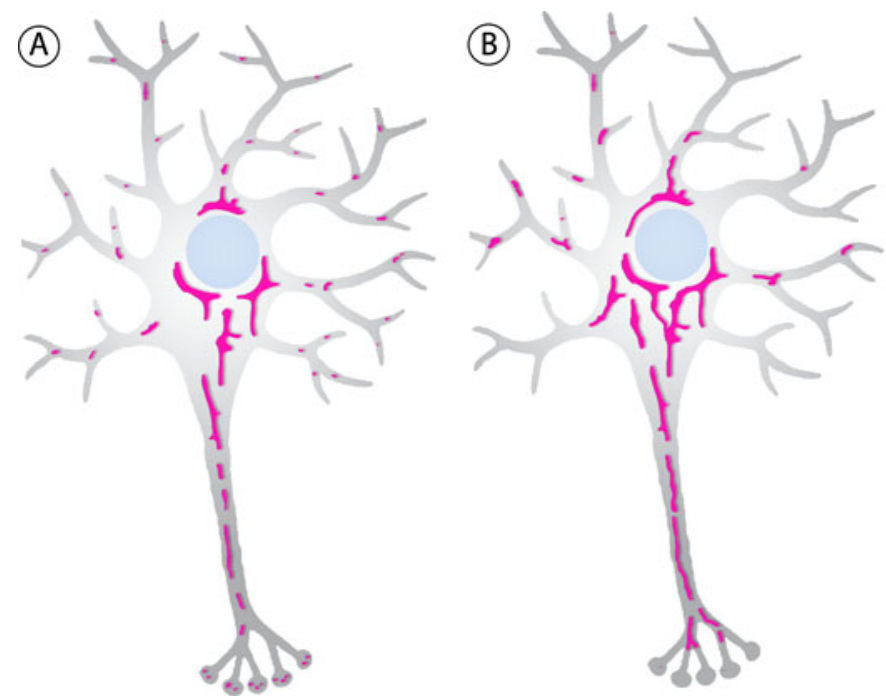

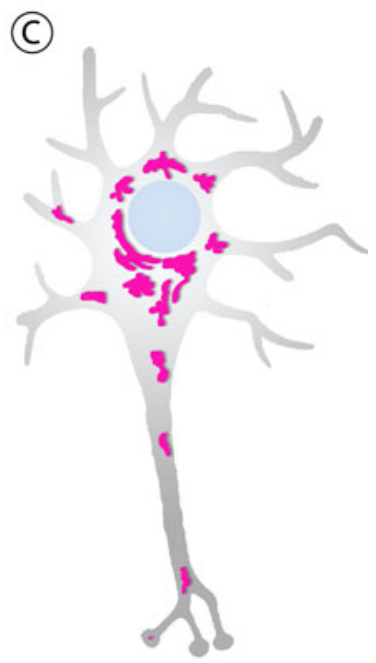

Fig. 3 Mitochondrial phenotypes in fission-incompetent neurons. a Different mitochondrial phenotypes have been reported for Drp1deficient neurons. Wildtype mitochondrial morphology is characterized by evenly distributed organelles in the axonal, neuritic and perikaryon compartments. b Following functional Drp1 inactivation in neurons an elongated mitochondrial morphology that branches out into the neurites can be observed; under these conditions,

devoid of lobar structures, and showed decreased numbers of Purkinje neurons containing fewer mitochondria. Ishihara et al. [45] ablated Drp1 pan-neurally using the nestin promoter, which normally gets activated at around day e9.5. At e18.5, a reduction of forebrain size with white matter hypoplasia and periventricular leukomalacia was observed, accompanied by markedly enlarged subdural and ventricular spaces. In addition, hypoplasia of brain stem and cerebellum was noted. Further analyses revealed a significantly increased number of TUNEL-positive neurons in deep cortical layers. Interestingly, Drp1 is highly expressed in layer $\mathrm{V}$ of the adult mouse neocortex [6], roughly correlating with the many TUNEL-positive cells at the cortex-white matter junction of Drp1-ablated mice [45]. On the cellular level, it is interesting to note that neurons express mitochondria-shaping proteins at particularly high levels, which very likely reflects the peculiar morphological and biochemical characteristics of this highly ATP-dependent cell type. In vitro, expression of the dominant-negative Drp1 mutant K38A in rat hippocampal neurons reduces the number of dendritic spines, underscoring again the importance of Drp1 for neuronal morphogenesis [56] (Fig. 3c). In line with these observations, mixed neuronal cultures derived from nestin-Cre Drp1 knockout mice also feature decreased synapse formation and exhibit a decreased number of neurites [45] (Fig. 3c), whereas in postnatal cortical mouse neurons Drp1 downregulation or expression of a dominant-negative Drp1 mutant causes cell death [104]. Interestingly, also mitochondria do not fragment during apoptosis and are protected from cell death $[4,18,21,24,46,63,101,119,120]$. $\mathbf{c}$ Others found impaired mitochondrial trafficking with elongated organelles aggregating within the perikaryon, accompanied by decreased numbers of neurites and synapses [45, 56, 104]. The relative contribution of potential mtDNA losses as well as ROS to neuronal degeneration has not been clarified in detail

activity-stimulated formation of excitatory synapses is blocked by expression of dominant-negative Drp1 mutants, whereas synapse formation is potentiated by the expression of wildtype Drp1 [56].

Finally, it should be noted here that synapses in Drosophila Drp1 mutants are largely devoid of mitochondria, apparently without synapse function impairments. However, significant defects in the ATP-dependent recruitment of reserve pool vesicles are found, indicating that mitochondria present in the synaptic compartment are required to satisfy sustained local energy needs [106].

Mitochondria are abundant at synapses [89] as well as within the active growth cones of developing neurons [70]. As will be discussed later, mitochondrial trafficking is an energy-dependent process that requires appropriate structure and function of the organelles. Mitochondria in neurons lacking functional Drp1 tend to aggregate in the perikarya [45, 56, 104] (Fig. 3c). In the developmental models, the neuronal demise that follows the inactivation of Drp1 seems to be largely dependent on impaired synapse formation and neurite outgrowth, both of which can probably, at least in part, be attributed to mitochondrial trafficking problems. Curiously, the mitochondrial phenotypes that can be observed in neurons with reduced Drp1 activity are not uniform, ranging between an elongated organelle network that branches out into neurites (Fig. 3b) to aggregated perinuclear mitochondrial accumulations (Fig. 3c). This phenotypic variability seems to depend on specific experimental conditions such as, e.g., the mode of 
functional Drp1 inactivation, time after transfection or lentiviral infection $[4,18,21,24,40,46,66,101,119$, 120]. It is thus tempting to speculate that the elongated mitochondrial morphology branching into neurites and dendrites (Fig. 3b) represents a transitory state that can be observed shortly after Drp1 functional inactivation, whereas the perinuclear mitochondrial clustering associated with degeneration of neuronal processes (Fig. 3c) may result from long-term Drp1 deprivation.

\section{Drp1 in Alzheimer's disease}

Clinically characterized by progressive decline of cognitive functions and memory, Alzheimer's disease (AD) is a lateonset neurodegenerative disease that leads to the most common form of dementia. Traditional pathological hallmark features of $\mathrm{AD}$ comprise the formation of neurofibrillar tangles composed of hyperphosphorylated tau protein as well as senile plaques formed by aggregated amyloidogenic A-beta-peptides derived from proteolytic cleavage of amyloid precursor protein (APP). During the past few years evidence has been emerging indicating that dysregulated mitochondrial structure and morphology may represent an important component in the complex pathogenesis of AD.

Neurons that are either overexpressing APP or exposed to toxic A-beta peptides display abnormal levels of mitochondria shaping proteins in association with a perinuclearly aggregated mitochondrial phenotype [4, 60, 113, 114]. These findings are paralleled by studies of $\mathrm{AD}$ patient brains reporting abnormal expression levels of mitochondrial morphogenic proteins [60, 113]. Of note, specific abnormal interactions between Drp1 and A-beta have recently been identified in AD brains [60]. In addition, Tau hyperphosphorylation as well as neurofibrillary tangle formation seem to be linked to an abnormal mitochondrial distribution in neurons [52]. Whether this abnormality exclusively results from disruption of microtubule-based organelle transport or whether dysbalanced mitochondrial fission and fusion processes contribute to this phenotype, remains unclear at present. Finally, specific post-translational Drp1 modifications have recently been implicated with AD. For example, increased levels of Drp1 phosphorylation at position S616 are found in AD brains [113]. Moreover, Drp1 can undergo S-nitrosylation at residue $\mathrm{C} 644$, promoting mitochondrial fission [19, 73, 113].

\section{Drp1 in Huntington's Chorea}

A progressive monogenic neurodegenerative disorder, Huntington's disease (HD) is inherited in an autosomal- dominant pattern. It is clinically characterized by involuntary movements such as chorea and dystonia, accompanied by psychiatric disturbances and cognitive decline leading to dementia. Albeit most of the patients experience initial symptoms in their midlife, HD also includes juvenile onset forms. On the molecular level, an intragenic CAG-triplett expansion within the huntingtin (Htt) gene on chromosome 4 results in an abnormal stretch of polyglutamine residues, determining age of onset and disease severity. Histopathological correlates include progressive neuronal loss affecting in particular striatal GABAergic spiny neurons, accompanied by atrophy and gliosis in basal ganglia, cortex, and hippocampus. With several theories existing on the neurotoxicity of mutant Htt aggregates, the exact pathobiology of HD remains to be determined. Transcriptional dysregulation, defective intracellular trafficking, and abnormal mitochondrial bioenergetics and dynamics are all currently being discussed as pathogenic factors [109].

HD-like phenotypes can easily be recapitulated in transgenic mice expressing full-length mutant $\mathrm{Htt}$ or a N-terminal Htt fragment. Neuronal losses and other pathological features of HD as well as the occurrence of clinical symptoms following pharmacologic inhibition of electron transport chain (ETC) complex II [8] lend strong support for a significant mitochondrial component in HD pathogenesis.

Recent studies in HD patient lymphoblasts as well as in primary striatal neurons isolated from HD mouse models revealed a fragmented mitochondrial morphology in concert with an increased susceptibility to apoptotic stimuli. Additionally, increased calcineurin activity paralleled by dephosphorylation and association of Drp1 with mitochondria has been reported [21]. Intriguingly, this phenotype is accompanied by changes in mitochondrial ultrastructure. Of note, the effects of mutant Htt on mitochondrial fragmentation can be rescued either by the expression of Opa1, the dominant-negative Drp1 mutation K38A, or a dominant-negative calcineurin mutant. The fact that, unlike Opa1, expression of the mitochondrial fusion protein Mfn1 is unable to counteract the increased apoptosis susceptibility of mutant Htt cells emphasizes the importance of Opa1-dependent cristae maintenance, which provides protection from inappropriate cytochrome $c$ release and ensuing apoptotic cell death [21].

In addition to increased mitochondrial fragmentation and neuronal cell death susceptibility, impaired mitochondrial trafficking has also been demonstrated in mutant Htt cell lines. Mutant huntingtin binds to mitochondria, where it assembles into large oligomers at prospective fission sites, similar to Drp1 complexes. Apparently, the co-localization of Drp1 and mutant Htt at mitochondrial fission sites is based on specific Drp1/Htt interactions, that 
are much weaker in the case of wildtype in comparison to mutated Htt [97].

In human HD brain, the occurrence of mutant $\mathrm{Htt}$ aggregates correlates well with the expression of mitochondrial fission-mediating proteins. In particular, in striatal and cortical specimens from HD patients, mitochondrial fission genes Drp1 and Fis1 are expressed at higher mRNA and protein levels, whereas Opa1, Mfn1 and Mfn2 fusion proteins are decreased. Finally, an increased expression of mitochondrially encoded ETC genes as well as significant oxidative DNA damage have been demonstrated in human HD brains, once again reflecting the pathogenically important relationship between mutant HTT and mitochondrial dysfunction in HD [95].

\section{Drp1 in Parkinson's disease}

Parkinson's disease (PD) is the second most common neurodegenerative disease affecting roughly $1 \%$ of the population aged 65 years or above [107]. Roughly $10 \%$ of all cases are associated with mutations in different PARK gene loci, the remaining cases being sporadic with age being the major risk factor. PD pathology is characterized by the loss of dopaminergic neurons in the substantia nigra pars compacta and the formation of intraneuronal inclusions known as Lewy bodies (LB) [30, 31]. On the molecular level, PD pathogenesis has long been regarded as being primarily caused by mitochondrial dysfunction, and, more recently, also by impaired protein turnover causing accumulation of toxic protein aggregates. Apart from LB formation, levels of core components of the ubiquitin-proteasome system are decreased [62] paralleled by increased numbers of autophagic vacuoles in PD brains [2]. Further, it has long been known that exposure to ETC complex I inhibiting toxins such as 1-methyl-4-phenylpiridinium $\left(\mathrm{MPP}^{+}\right)$, rotenone or paraquat can rapidly induce PD-like symptoms in humans and laboratory animals [54, 65]. In addition, complex I deficiency [91] and elevated ROS levels [61] are frequently observed in PD patients. Moreover, two of the PARK genes, parkin and Pink1, play pivotal roles in the removal of damaged mitochondrial organelles via mitophagy [75, 76], and work published earlier this year has identified Drp1 as one of the targets of the E3 ubiquitin ligase, parkin [111].

Interestingly, expression of PKA or of the Drp1 S637D phosphomimetic mutation (this S637 phosphorylation is mediated by PKA) can revert the mitochondrial profission phenotype of Pink1-deficient cells [23]. In addition, Pink1-deficient cells display increased calcineurin activity; accordingly, their pro-fission phenotype can be reverted by treatment with the calcineurin inhibitor FK506 [90].
The fascinating interplay of parkin, Pink1, and Drp1, which orchestrates mitophagy will be discussed in greater detail by Pilsl and Winklhofer (also this issue). Suffice it to note here that alpha-synuclein, another PARK gene and major LB component [98], associates with mitochondria and induces Drp1-independent mitochondrial fragmentation [72]. It will be interesting to test in how far recently reported physiological tetrameric alpha-synuclein [5] is capable of modulating mitochondrial dynamics. In summary, mitochondrial dysfunction significantly contributes to PD pathology. To what extent dysregulated mitochondria morphogenesis and turnover contribute to the pathogenesis of sporadic PD remains to be elucidated.

\section{Drp1 in ischemic brain damage and excitotoxicity}

Surrounding the necrotic ischemic core where neurons perish by necrosis as an immediate consequence of lacking nutrients, the penumbra receives reduced nutrient supply and remains metabolically active. Cells within the penumbra are susceptible to apoptotic cell death, which occurs primarily as a consequence of excitatory amino acids and proteolytic enzymes released from the necrotic core region. As the penumbra can make up more than half of the total infarction size and apoptotic pathways can be targeted increasingly successfully [11], understanding the pathophysiology of ischemic brain damage is imperative for the successful development of neuroprotective strategies targeting such socio-economically important conditions as ischemic stroke [11].

In murine models of cerebral ischemia mitochondria within the penumbra have been shown to fragment [4]. As Drp1-mediated mitochondrial fragmentation is commonly observed early during apoptosis with its inhibition delaying programmed cell death in most experimental scenarios, chemical inhibition of Drp1 using small molecule inhibitors has recently emerged as a promising therapeutic strategy. This is exemplified by mdivi-1, a Drp1-inhibiting compound [12] which significantly increases neuronal survival in a murine retinal ganglion ischemia model [82]. Remarkably, mdivi-1 is also effective in reducing infarct sizes in renal [10] and cardiac ischemia [77]. In this context, it is interesting to note that transgenic mice overexpressing miR-499 show reduced myocardial infarction sizes when subjected to cardiac ischemia [112]. miR-499 is a micro RNA targeting calcineurin (which under normal conditions favors mitochondrial fragmentation via Drp1 dephosphorylation [14]).

In addition to increasing susceptibility toward apoptosis, the pronounced lack of nutrients within the penumbra may be sufficient to trigger adaptive autophagic responses. The observation that autophagy seems to decline with age [57] 
might represent one underlying biochemical correlate for the relatively poor neurologic outcome of older ischemic stroke patients. Mounting evidence suggests that, in addition to fostering the elimination of potentially toxic protein aggregates, autophagy can serve to maintain high ATP concentrations. Autophagy may thus be fundamentally important under conditions of limited nutrient supply as reflected by the observation that during starvation-one of the traditional autophagy triggers-mitochondria become hyperfused [38]. Such hyperfused states facilitate the formation of ATP synthase dimers, which are more efficient in producing ATP [99]. As fragmented mitochondria are less potent ATP producers, improving energetic levels within the penumbra by pharmacologically inhibiting mitochondrial fission may represent a neuroprotective strategy remaining to be tested by future experimentation.

In addition to the limited nutrient supply, neurons within in the cerebral penumbra are exposed to extreme $\mathrm{Ca}^{2+}$ mediated excitotoxic stress. Energy depletion inhibits membrane potential maintenance with the subsequent depolarization triggering the release of synaptic vesicles that contain excitatory amino acids. Moreover, excitotoxic stress induced by activation of ionotropic NMDA receptors on presynaptic membranes allows for $\mathrm{Ca}^{2+}$ influx, thereby dramatically elevating intracellular $\mathrm{Ca}^{2+}$ levels whichwhen exceeding the uptake capacities of the endoplasmic reticulum-may result in neuronal cell death. Sharp rises in mitochondrial $\mathrm{Ca}^{2+}$ can activate Drp1 through calcineurinmediated dephosphorylation, and trigger mitochondrial permeability transition with subsequent activation of the mitochondrial gateway to apoptosis. In addition, $\mathrm{Ca}^{2+}$ influx into neurons is known to activate calpains (a class of calcium-dependent cysteine proteases). Calpains have been shown to stimulate neuron-specific cyclin-dependent kinase $5(\mathrm{Cdk} 5)$ forcing cell cycle re-entry of postmitotic neurons and promoting neuronal death [11, 59]. Moreover, glutamate stimulation of neuronal NMDA receptors leads to reduced mitochondrial motility and fragmentation of the organelles, finally culminating in cell death [47, 87, 119]. Of note, death induced by NMDA receptor-stimulated $\mathrm{Ca}^{2+}$ influx can be blocked by overexpression of Opa1 [47] and, in vivo, by overexpression of the endogenous calpain inhibitor, calpastatin [42].

Taken together, the currently available evidence suggests that Drp1-mediated mitochondrial fission contributes to neuronal cell loss under ischemic and excitotoxic conditions. Pharmacological inhibition of the fission machinery, e.g., by the small molecule Drp1 inhibitor mdivi-1, appears to represent a plausible approach that should extensively be tested for its potential as neuroprotective therapeutic strategy. Of note, the protective effects of chemical Drp1 inhibition under ischemic conditions may - at least in part-be attributed to metabolic effects associated with mitochondrial elongation rather than inhibition of apoptosis per se.

\section{Drp1 and metabolism}

Although there is no robust experimental data available to verify a link between Drp1 activity (or organelle morphology) and mitochondrial respiration in neurons, recent evidence indicates that hyperfused mitochondria are more efficient in ATP production [38, 67, 102]. This hyperfused state is observed under stress conditions such as exposure to toxins (UV light, cycloheximide, actinomycin D) [102], during starvation [38], or during the energy-demanding G1 to $\mathrm{S}$ phase transition of mitosis [67]. Of note, the starvation-induced hyperfused state depends on PKA-mediated phosphorylation of Drp1 at Ser637 that inactivates Drp1 fission activity. In addition, ATP synthase dimer formation is increased [38] resulting in more efficient ATP generation under low mitochondrial membrane potential conditions [99]. In how far ATP-Synthase dimer formation leading to increased mitochondrial ATP production by hyperfused mitochondria represents a general protective mechanism in stressed cells remains to be elucidated. Long-term genetic Drp1 ablation, on the other hand, decreases respiratory activity [81, 84, 103] or has no to negligible effects [45]. Perhaps, genetic Drp1 deletion induces additional compensatory mechanisms beyond just tipping the balance toward mitochondrial fusion. Nevertheless, forced induction of hyperfusion through direct inhibition of mitochondrial fission may potentially represent a neuroprotective strategy for acute neuronal stress conditions such as ischemia, deserving further exploration.

\section{Drp1 and impairment of mitochondrial quality control}

As mitochondrial quality control mechanisms are reviewed elsewhere in this issue, we will focus our discussion here to the question of how Drp1 relates to mitochondrial quality surveillance.

Three mitochondrial components require continuous recycling: mtDNA, proteins, and lipids. Single mitochondrial organelles carry several mtDNA copies, ensuring that intact mtDNA molecules can compensate for damaged (i.e., mutated) ones. It is thus believed that-via fusion of the organelles-mitochondria constantly exchange their mtDNA content within single cells. Proof of functionally important mtDNA exchange was first provided more than a decade ago through a very elegant approach [78]: through hybridizing two cell lines each carrying one specific mtDNA mutation associated with respiratory deficiency, normal respiration rates could be restored [78]. In addition, 
experiments with mice carrying mtDNA mutations proved that, in fact, a population of mitochondria within one cell shares a common pool of mtDNA [71]. The process of mitochondrial complementation is highly dependent on organelle fusion as demonstrated in a murine muscle-specific Mfn2 knockout model, where mtDNA mutations accumulate at similar rates as in mice expressing a proofreading defective mitochondrial DNA polymerase mutant [16]. Evidence suggesting a significant role for Drp1mediated mitochondrial fission in mtDNA maintenance is scarce; in HeLa cells, Drp1 downregulation leads to loss of mtDNA as determined by immunofluorescence microscopy using anti-DNA antibodies [84].

The mechanisms of mitochondrial protein and lipid turnover are still poorly understood at present. Through a specialized autophagic process termed mitophagy, doublemembrane structures are formed which enclose entire mitochondrial organelles and which subsequently fuse with lysosomes. It has been shown that the pro-fission OMM protein Fis1 is essential for mitochondrial trafficking to autophagosomes [39]. Of note, expression of mutant Drp1 is able to block mitochondria removal [3]. More recently, it has been shown that Drp1-mediated fission can give rise to depolarized single mitochondrial units that subsequently undergo mitophagy [103]. In line with this fundamental observation, Drp1 ablation leads to an accumulation of oxidized proteins in mitochondria [84, 103]. Finally, as mentioned earlier, the Pink1/parkin pathway that also involves direct interactions with Drp1, contributes significantly to mitochondrial quality control [111]. As, at the moment, no assays are available to directly quantify mitochondrial turnover and mitophagy, the direct contributions of Pink1/parkin and Drp1 to mitochondria quality control via organelle turnover/mitophagy still remain to be clarified in detail.

\section{Drp1 and aberrant mitochondrial trafficking}

Neurons, the longest human cells which can exceed lengths of one meter, are especially dependent on a proper mitochondrial distribution. Distinct long- and short-distance mitochondrial transport mechanisms exist in order to satisfy the particularly high local energy demands at synapses and nodes of Ranvier. Significantly, aberrant mitochondrial trafficking is likely the primary cause underlying the CNS developmental defects including perturbed synapse formation in Drp1 knockout mice. In neurons, microtubule tracks are used as long-distance transport rails to cargo membrane vesicles and cellular organelles including mitochondria. Axonal transport of mitochondria is bidirectional and thought to be coordinated with neuronal outgrowth [70]. Whereas anterograde (i.e., synapse-bound) cargo movement is mediated by kinesins, dynein/dynactin act promiscuously in retrograde (i.e., perikaryon-bound) organelle transport.

The protein couple Miro/Milton, acting in concert with kinesins, is responsible for anterograde mitochondrial trafficking, with $\mathrm{Ca}^{2+}$ binding Miro being anchored to the OMM. In a calcium-unbound state, Miro binds to Milton, which in turn can bind to kinesin motorproteins. Upon exposure to high calcium levels, as they occur, e.g., within the pre- or postsynaptic compartments, the molecular Miro/ Milton interaction gets disrupted, releasing mitochondria [58]. The only mitochondria-shaping protein so far directly implicated in this process is Mfn2, which interacts with the Miro/Milton complex.

The multimeric dynein complex comprising dynactin and its subunit p50/dynamitin is responsible for retrograde mitochondrial transport. Interestingly, mitochondrial Drp1 foci colocalize with microtubules [105, 118]. Dysruption of the dynein complex or expression of the Drp1 K38A mutant interrupts Drp1/microtubule colocalization thereby blocking mitochondrial transport [105]. Collectively, the available evidence strongly points to a co-dependence of Drp1 and dynein for long-distance mitochondrial trafficking, which is of obvious importance in highly polarized neurons.

Short-distance mitochondrial transport is mediated by actin filaments. In analogy to the disruption of dynein complexes, disruption of actin filaments has been shown to attenuate mitochondrial Drp1 translocation and organelle fission [26].

\section{Drp1 in apoptosis}

Soon after Drp1's discovery as a mitochondrial fission protein, it was observed that the mitochondrial fragmentation occurring during apoptosis could be inhibited by overexpression of the dominant-negative Drp1 mutant K38A. Blocking Drp1 function not only inhibited fragmentation of the organelles but, more importantly, also delayed mitochondrial cytochrome $c$ release and subsequent apoptotic cell death [35]. However, the general necessity of mitochondrial fission for apoptosis to proceed remains a matter of debate largely attributable to specific experimental setups (including cell lines, specific stimuli, differential sensitivity of various cell death detection assays, as well as different means of Drp1 functional inactivation) [49, 94, 116].

We would like to restrict our discussion here to comment on the differential results reported for the available Drp1 knockout mouse models [45, 108]. Whereas Wakabayashi et al. [108] using non-immortalized mouse embryonic fibroblasts in their cell death assays, did not 
observe a delay in apoptosis, the Mihara group using immortalized fibroblasts were able to show just that [45]. Interestingly, complete Drp1 ablation did not block, yet delayed mitochondrial fragmentation during apoptosis [45]. Perhaps, this effect is attributable to compensatory changes in expression levels of other mitochondria-shaping proteins, which are already altered at steady state in Drp1 null fibroblasts [45]. In summary, genetic Drp1 ablation delays programmed cell death but not sufficient to block it.

On the organelle level, Drp1 has been proposed to facilitate apoptotic cell death by impinging either on outer mitochondrial membrane permeabilization or, alternatively, on mitochondrial cristae remodeling. Key mediators of OMM permeabilization are the "multidomain proapoptotics" Bax and Bak. Both proteins belong to the Bcl-2 family of proteins and get activated in the OMM by socalled "BH3-only" proteins, a Bcl-2 family subset. Once activated, mitochondria release proapoptotic factors such as cytochrome $c$ into the cytosol, where subsequent activation of downstream effector caspases occurs via formation of the apoptosome, a specialized protein complex containing APAF1 and pro-caspase-9 [116]. Of note, in healthy cells, about $85 \%$ of the soluble cytochrome $c$ pool resides within the cristae compartment, made up by specialized inner mitochondrial membrane structures. During apoptosis, dramatic morphological changes termed cristae remodeling take place, facilitating the release of cytochrome $c$ that takes place during programmed cell death [7, 93]. Expression of the dominant-negative Drp1 mutant K38A can rescue cristae remodeling induced by overexpression of the proapoptotic Bcl-2 protein Bik [37] as well as by expression of mutant huntingtin [21]. As to now, a plausible answer on how a cytosolic protein such as Drp1 that anchors to the OMM, can influence cristae remodeling, is lacking. Indirect effects on the proteolytic processing of Opa1, a key regulator of cristae morphology, may represent a plausible scenario as, in fact, levels of proteolytically derived Opa1 isoforms are altered in several Drp1-ablated cell models [45, 69].

During apoptosis, Bax translocation from the cytosol to the OMM is dependent on Drp1 and facilitated by cardiolipin, a mitochondrial lipid residing in the IMM. It has been postulated that, in addition to modulate cristae modeling, Drp1 is involved in the formation of hemifusion sites that bring inner and outer mitochondrial membranes into close contact with each other, thereby enabling the exchange of lipids between the two membranes which facilitates Bax membrane integration [68]. This mechanism could be particularly important in neurons, where nitric oxide donor-induced translocation of Bax to mitochondria is Drp1 dependent [120]. Of note, the neuronal death in this model requires Drp1 and Bax but no caspase activation [120], exemplifying a mode of neuronal damage engaging certain modules of the molecular apoptosis cascade without following the complete pathway of classical intrinsic programmed cell death.

Neuronal apoptosis related to changes in mitochondrial morphology has poorly been investigated so far. It is generally difficult to detect in vivo as cells dying by apoptosis are swiftly removed by microglia [74]. Drp1 knockout neurons, which display an aggregated mitochondrial morphology, are more sensitive to the pan-kinase inhibitor staurosporine (STS) as well as to ceramide, a $\mathrm{Ca}^{2+}$ stress-inducing drug [45].

In contrast, neurons transiently deprived of functional Drp1 display an elongated mitochondrial phenotype (Fig. 3b) and show resistance to various apoptotic stimuli such as STS [21, 66, 119], rotenone [4, 24], $\mathrm{MPP}^{+}$[4], A-beta peptide 25-35 [4], nitric oxide [4, 120], hydrogen peroxide [18, 46], and metamphetamine [101] (Fig. 4). The discrepancy between these conditions of functional Drp1 inactivation with respect to cell death susceptibility may, as already discussed, be related to the timing of Drp1 ablation: whereas long-term Drp1 ablation favors neuronal death, short-term deletion seems to exert neuroprotective effects. The lack of mitochondrial fission under conditions of genetic Drp1 ablation is likely compensated for by the degradation of the Opa1 protein along with downregulated expression of Mfn1 and Mfn2, all of which result in decreased fusion of the organelles [45]. Interestingly, in AD brains, Mfn1, Mfn2, Opa1 and Drp1 are simultaneously downregulated and this state seems to be associated with perinuclear mitochondrial aggregation and decreased numbers of dendrites and synapses [113].

$\mathrm{Ca}^{2+}$ influx has already been discussed as an important inducer of neuronal death. In the context of apoptosis, increased mitochondrial $\mathrm{Ca}^{2+}$ uptake can trigger mitochondrial permeability transition, organelle swelling with rupture of the OMM, culminating in the release of proapoptotic factors into the cytosol. In addition, $\mathrm{Ca}^{2+}$ activates calpains which trigger apoptosis by cleavage of the BH3only protein Bid and by processing of the Cdk5 activator p35 to yield the more active p25 form [59]. When activated, Cdk5, which is highly expressed in postmitotic neurons, mediates STS-induced caspase-dependent neuronal death that is preceded by mitochondrial fragmentation [66]. In summary, for neurons, currently available evidence suggests a tight interplay between pathways of $\mathrm{Ca}^{2+}$-mediated neuronal death and classical apoptosis that in both cases involves dysregulated mitochondrial dynamics (Fig. 4).

\section{Therapeutic targeting of mitochondria}

Most avenues for therapeutic targeting of mitochondria have so far been focused on counteracting ROS production 


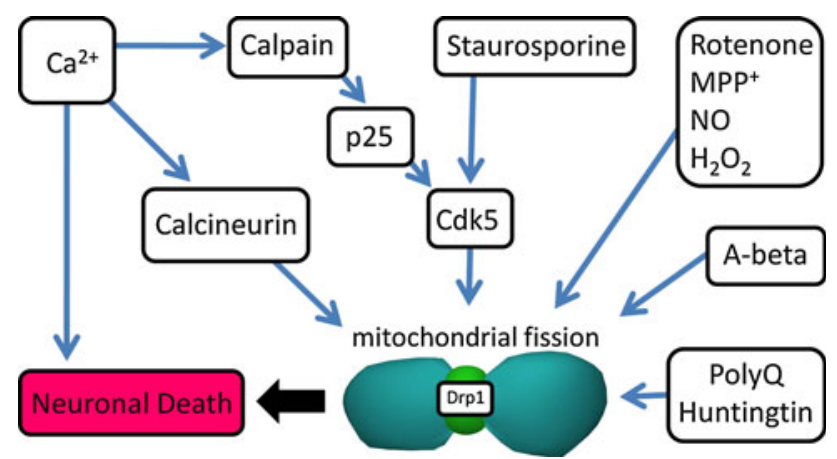

Fig. 4 Mitochondrial fission in neuronal death. Mitochondrial fission has been implicated in the pathogenesis of several neurodegenerative diseases. Compounds mimicking neurotoxicity as it occurs in neurodegeneration have in vitro been shown to mediate neuronal death dependent on mitochondrial fission. Interestingly, Cdk5, implicated in the pathogenesis of several neurodegenerative conditions, functions as an upstream regulator of mitochondrial fission during neuronal apoptosis. The protein also plays a prominent role in $\mathrm{Ca}^{2+}$-mediated cell death pathways. Here, $\mathrm{Ca}^{2+}$-activated calpains process the p35 Cdk5 activator protein to yield p25, resulting in enhanced Cdk5 activity. In addition, $\mathrm{Ca}^{2+}$ can also directly trigger mitochondrial fragmentation by calcineurin-mediated Drp1 S637. On the organelle level, intramitochondrial $\mathrm{Ca}^{2+}$ accumulation may lead to organelle swelling, opening of the permeability transition pore, and OMM rupture, culminating in the release of proapoptotic factors such as cytochrome $c$ from the mitochondria into the cytosol

by directing antioxidant molecules to the organelles [20]. For some CNS pathologies with underlying defects in mitochondrial function or energy production, such as ischemia, creatine, which is already approved for the adjuvant treatment of various myopathies, may be a promising candidate. Creatine has long been shown to increase mitochondrial respiration [110] and to promote neurite formation in vitro [56]. Furthermore, in rodent models of brain ischemia, creatine administration reduces infarct volumes [85]. In addition to this more "traditional" approach, tinkering chemically with the mitochondrial fusion/fission machinery seems to have great therapeutic potential through modulating organelle function. As discussed, genetic Drp1 ablation during neuronal development dramatically blocks synapse and neurite formation $[45,56]$ and Drp1 ablation in differentiated cultured neurons can even lead to cell death [104]. On the other hand, mitochondrial fission, mediated by Drp1, favors neuronal apoptosis [68] and toxin-mediated neuronal death [4, 21, $24,66,101,119,120]$ while inhibiting the formation of protective, metabolically more efficient hyperfused mitochondrial networks [38]. Hence, transient blockage of organelle fission during acute situations such as ischemic stroke may represent a veritable strategy to counteract neuronal damage. In addition, pharmacological modulation of mitochondrial fission in Huntington's disease where neurodegenerative pathology seems to directly result from imbalanced mitochondrial fusion and fission events, might represent another plausible therapeutic strategy. So far, all known small molecule compounds that modify mitochondrial morphology impinge on Drp1 either directly as is the case for mdivi-1 [12], or indirectly via inhibition of calcineurin, which can be achieved by FK506 (already FDA approved as immunosuppressant) or by PPD1 [13, 14]. Significantly, mdivi- 1 has already proven its efficacy in several rodent ischemia models [10, 12, 77, 82], whereas FK506 has only recently shown in vitro efficacy in protecting murine huntingtin knockin neurons from mild apoptotic stimuli [88].

Targeting of calpains and Cdk5 represents an effective strategy to inhibit $\mathrm{Ca}^{2+}$-mediated neuronal death upstream of mitochondrial fragmentation. A variety of calpain inhibitors have already proven to be protective in animal stroke models. However, non-selective calpain isoform inhibition has so far hampered respective clinical trials [29]. (S)-roscovitine, a novel Cdk5 inhibitor, has recently been tested successfully in a mouse stroke model, where it significantly reduced ischemic infarct size [64].

In summary, targeting mitochondria, either via modulating the balance between mitochondrial fission and fusion events or by interfering with $\mathrm{Ca}^{2+}$-induced cell death pathways, represents a novel strategy to counteract neuronal cell death. Especially, in acute situations such as ischemic stroke, modulating mitochondrial dynamics by inhibition of Drp1-driven mitochondrial fragmentation seems to be a promising therapeutic approach.

\section{Summary and perspective}

Since the concept of dysregulated mitochondrial dynamics has been introduced to the readers of Acta Neuropathologica in 2006 [34], we have seen a tremendous extension of our knowledge on the molecular mechanisms underlying mitochondrial fission and fusion processes. Moreover, it is now generally accepted that the dysregulation of mitochondrial dynamics is either causative or at least a significant factor in the pathogenesis of a spectrum of human disorders such as stroke, obesity, diabetes mellitus as well as neurodegenerative diseases. A detailed understanding of how dysregulated mitochondrial morphology leads to neuronal damage is a prerequisite to successfully interfere with mitochondria-driven neurodegenerative pathways. The recent development of small molecule inhibitors capable of counteracting inappropriately activated mitochondrial fission may represent a promising neuroprotective strategy. 


\section{References}

1. Alexander C, Votruba M, Pesch UE, Thiselton DL, Mayer S, Moore A, Rodriguez M, Kellner U, Leo-Kottler B, Auburger G, Bhattacharya SS, Wissinger B (2000) OPA1, encoding a dynamin-related GTPase, is mutated in autosomal dominant optic atrophy linked to chromosome 3q28. Nat Genet 26(2):211-215

2. Anglade P, Vyas S, Javoy-Agid F, Herrero MT, Michel PP, Marquez J, Mouatt-Prigent A, Ruberg M, Hirsch EC, Agid Y (1997) Apoptosis and autophagy in nigral neurons of patients with Parkinson's disease. Histol Histopathol 12(1):25-31

3. Arnoult D, Rismanchi N, Grodet A, Roberts RG, Seeburg DP, Estaquier J, Sheng M, Blackstone C (2005) Bax/Bak-dependent release of DDP/TIMM8a promotes Drp1-mediated mitochondrial fission and mitoptosis during programmed cell death. Curr Biol 15(23):2112-2118

4. Barsoum MJ, Yuan H, Gerencser AA, Liot G, Kushnareva Y, Graber S, Kovacs I, Lee WD, Waggoner J, Cui J, White AD, Bossy B, Martinou JC, Youle RJ, Lipton SA, Ellisman MH, Perkins GA, Bossy-Wetzel E (2006) Nitric oxide-induced mitochondrial fission is regulated by dynamin-related GTPases in neurons. EMBO J 25(16):3900-3911

5. Bartels T, Choi JG, Selkoe DJ (2011) Alpha-synuclein occurs physiologically as a helically folded tetramer that resists aggregation. Nature 477(7362):107-110

6. Belgard TG, Marques AC, Oliver PL, Abaan HO, Sirey TM, Hoerder-Suabedissen A, Garcia-Moreno F, Molnar Z, Margulies EH, Ponting CP (2011) A transcriptomic atlas of mouse neocortical layers. Neuron 71(4):605-616

7. Bernardi P, Azzone GF (1981) Cytochrome $c$ as an electron shuttle between the outer and inner mitochondrial membranes. J Biol Chem 256(14):7187-7192

8. Bouillet L, Cattoen C, Keilani A, Chatelain C, Mazars E (1998) Evaluation of a dot-blot technique for the detection of EpsteinBarr virus antibodies (IgG anti-viral capsid antigen). Ann Biol Clin 56(1):79-83

9. Braschi E, Zunino R, McBride HM (2009) MAPL is a new mitochondrial SUMO E3 ligase that regulates mitochondrial fission. EMBO Rep 10(7):748-754

10. Brooks C, Wei Q, Cho SG, Dong Z (2009) Regulation of mitochondrial dynamics in acute kidney injury in cell culture and rodent models. J Clin Invest 119(5):1275-1285

11. Broughton BR, Reutens DC, Sobey CG (2009) Apoptotic mechanisms after cerebral ischemia. Stroke 40(5):e331-e339

12. Cassidy-Stone A, Chipuk JE, Ingerman E, Song C, Yoo C, Kuwana T, Kurth MJ, Shaw JT, Hinshaw JE, Green DR, Nunnari J (2008) Chemical inhibition of the mitochondrial division dynamin reveals its role in Bax/Bak-dependent mitochondrial outer membrane permeabilization. Dev Cell 14(2):193-204

13. Cereghetti GM, Costa V, Scorrano L (2010) Inhibition of Drp1-dependent mitochondrial fragmentation and apoptosis by a polypeptide antagonist of calcineurin. Cell Death Differ 17(11):1785-1794

14. Cereghetti GM, Stangherlin A, Martins de Brito O, Chang CR, Blackstone C, Bernardi P, Scorrano L (2008) Dephosphorylation by calcineurin regulates translocation of Drp1 to mitochondria. Proc Natl Acad Sci USA 105(41):15803-15808

15. Chen H, Detmer SA, Ewald AJ, Griffin EE, Fraser SE, Chan DC (2003) Mitofusins Mfn1 and Mfn2 coordinately regulate mitochondrial fusion and are essential for embryonic development. J Cell Biol 160(2):189-200

16. Chen H, McCaffery JM, Chan DC (2007) Mitochondrial fusion protects against neurodegeneration in the cerebellum. Cell 130(3):548-562
17. Chen H, Vermulst M, Wang YE, Chomyn A, Prolla TA, McCaffery JM, Chan DC (2010) Mitochondrial fusion is required for mtDNA stability in skeletal muscle and tolerance of mtDNA mutations. Cell 141(2):280-289

18. Cheung EC, McBride HM, Slack RS (2007) Mitochondrial dynamics in the regulation of neuronal cell death. Apoptosis 12(5):979-992

19. Cho DH, Nakamura T, Fang J, Cieplak P, Godzik A, Gu Z, Lipton SA (2009) S-nitrosylation of Drp1 mediates beta-amyloid-related mitochondrial fission and neuronal injury. Science 324(5923): 102-105

20. Cocheme HM, Kelso GF, James AM, Ross MF, Trnka J, Mahendiran T, Asin-Cayuela J, Blaikie FH, Manas AR, Porteous CM, Adlam VJ, Smith RA, Murphy MP (2007) Mitochondrial targeting of quinones: therapeutic implications. Mitochondrion 7(Suppl):S94-S102

21. Costa V, Giacomello M, Hudec R, Lopreiato R, Ermak G, Lim D, Malorni W, Davies KJ, Carafoli E, Scorrano L (2010) Mitochondrial fission and cristae disruption increase the response of cell models of Huntington's disease to apoptotic stimuli. EMBO Mol Med 2(12):490-503

22. Cribbs JT, Strack S (2007) Reversible phosphorylation of Drp1 by cyclic AMP-dependent protein kinase and calcineurin regulates mitochondrial fission and cell death. EMBO Rep 8(10):939-944

23. Dagda RK, Gusdon AM, Pien I, Strack S, Green S, Li C, Van Houten B, Cherra SJ 3rd, Chu CT (2011) Mitochondrially localized PKA reverses mitochondrial pathology and dysfunction in a cellular model of Parkinson's disease. Cell Death Differ 18(12):1914-1923

24. Dagda RK, Merrill RA, Cribbs JT, Chen Y, Hell JW, Usachev YM, Strack S (2008) The spinocerebellar ataxia 12 gene product and protein phosphatase $2 \mathrm{~A}$ regulatory subunit Bbeta 2 antagonizes neuronal survival by promoting mitochondrial fission. J Biol Chem 283(52):36241-36248

25. de Brito OM, Scorrano L (2008) Mitofusin 2: a mitochondriashaping protein with signaling roles beyond fusion. Antioxid Redox Signal 10(3):621-633

26. De Vos KJ, Allan VJ, Grierson AJ, Sheetz MP (2005) Mitochondrial function and actin regulate dynamin-related protein 1-dependent mitochondrial fission. Curr Biol 15(7):678-683

27. Delettre C, Lenaers G, Griffoin JM, Gigarel N, Lorenzo C, Belenguer P, Pelloquin L, Grosgeorge J, Turc-Carel C, Perret E, Astarie-Dequeker C, Lasquellec L, Arnaud B, Ducommun B, Kaplan J, Hamel CP (2000) Nuclear gene OPA1, encoding a mitochondrial dynamin-related protein, is mutated in dominant optic atrophy. Nat Genet 26(2):207-210

28. Detmer SA, Vande Velde C, Cleveland DW, Chan DC (2008) Hindlimb gait defects due to motor axon loss and reduced distal muscles in a transgenic mouse model of Charcot-Marie-Tooth type 2A. Hum Mol Genet 17(3):367-375

29. Donkor IO (2011) Calpain inhibitors: a survey of compounds reported in the patent and scientific literature. Expert Opin Ther Pat 21(5):601-636

30. Ehringer H, Hornykiewicz O (1960) Distribution of noradrenaline and dopamine (3-hydroxytyramine) in the human brain and their behavior in diseases of the extrapyramidal system. Klin Wochenschr 38:1236-1239

31. Fearnley JM, Lees AJ (1991) Ageing and Parkinson's disease: substantia nigra regional selectivity. Brain 114(Pt 5):2283-2301

32. Figueroa-Romero C, Iniguez-Lluhi JA, Stadler J, Chang CR, Arnoult D, Keller PJ, Hong Y, Blackstone C, Feldman EL (2009) SUMOylation of the mitochondrial fission protein Drp1 occurs at multiple nonconsensus sites within the B domain and is linked to its activity cycle. Faseb J 23(11):3917-3927 
33. Ford MG, Jenni S, Nunnari J (2011) The crystal structure of dynamin. Nature 477(7366):561-566

34. Frank S (2006) Dysregulation of mitochondrial fusion and fission: an emerging concept in neurodegeneration. Acta Neuropathol 111(2):93-100

35. Frank S, Gaume B, Bergmann-Leitner ES, Leitner WW, Robert EG, Catez F, Smith CL, Youle RJ (2001) The role of dynaminrelated protein 1, a mediator of mitochondrial fission, in apoptosis. Dev Cell 1(4):515-525

36. Friedman JR, Lackner LL, West M, Dibenedetto JR, Nunnari J, Voeltz GK (2011) ER tubules mark sites of mitochondrial division. Science 334(6054):358-362

37. Germain M, Mathai JP, McBride HM, Shore GC (2005) Endoplasmic reticulum BIK initiates DRP1-regulated remodelling of mitochondrial cristae during apoptosis. EMBO J 24(8):15461556

38. Gomes LC, Di Benedetto G, Scorrano L (2011) During autophagy mitochondria elongate, are spared from degradation and sustain cell viability. Nat Cell Biol 13(5):589-598

39. Gomes LC, Scorrano L (2008) High levels of Fis1, a pro-fission mitochondrial protein, trigger autophagy. Biochim Biophys Acta 1777(7-8):860-866

40. Han XJ, Lu YF, Li SA, Kaitsuka T, Sato Y, Tomizawa K, Nairn AC, Takei K, Matsui H, Matsushita M (2008) CaM kinase Ialpha-induced phosphorylation of Drp1 regulates mitochondrial morphology. J Cell Biol 182(3):573-585

41. Harder Z, Zunino R, McBride H (2004) Sumo1 conjugates mitochondrial substrates and participates in mitochondrial fission. Curr Biol 14(4):340-345

42. Higuchi M, Tomioka M, Takano J, Shirotani K, Iwata N, Masumoto H, Maki M, Itohara S, Saido TC (2005) Distinct mechanistic roles of calpain and caspase activation in neurodegeneration as revealed in mice overexpressing their specific inhibitors. J Biol Chem 280(15):15229-15237

43. Ingerman E, Perkins EM, Marino M, Mears JA, McCaffery JM, Hinshaw JE, Nunnari J (2005) Dnm1 forms spirals that are structurally tailored to fit mitochondria. J Cell Biol 170(7):1021-1027

44. Ishihara N, Eura Y, Mihara K (2004) Mitofusin 1 and 2 play distinct roles in mitochondrial fusion reactions via GTPase activity. J Cell Sci 117(Pt 26):6535-6546

45. Ishihara N, Nomura M, Jofuku A, Kato H, Suzuki SO, Masuda K, Otera H, Nakanishi Y, Nonaka I, Goto Y, Taguchi N, Morinaga H, Maeda M, Takayanagi R, Yokota S, Mihara K (2009) Mitochondrial fission factor Drp1 is essential for embryonic development and synapse formation in mice. Nat Cell Biol 11(8):958-966

46. Jahani-Asl A, Cheung EC, Neuspiel M, MacLaurin JG, Fortin A, Park DS, McBride HM, Slack RS (2007) Mitofusin 2 protects cerebellar granule neurons against injury-induced cell death. J Biol Chem 282(33):23788-23798

47. Jahani-Asl A, Pilon-Larose K, Xu W, MacLaurin JG, Park DS, McBride HM, Slack RS (2011) The mitochondrial inner membrane GTPase, optic atrophy 1 (Opa1), restores mitochondrial morphology and promotes neuronal survival following excitotoxicity. J Biol Chem 286(6):4772-4782

48. James DI, Parone PA, Mattenberger Y, Martinou JC (2003) hFis1, a novel component of the mammalian mitochondrial fission machinery. J Biol Chem 278(38):36373-36379

49. Karbowski M (2010) Mitochondria on guard: role of mitochondrial fusion and fission in the regulation of apoptosis. Adv Exp Med Biol 687:131-142

50. Karbowski M, Neutzner A, Youle RJ (2007) The mitochondrial E3 ubiquitin ligase MARCH5 is required for Drp1 dependent mitochondrial division. J Cell Biol 178(1):71-84
51. Kashatus DF, Lim KH, Brady DC, Pershing NL, Cox AD, Counter CM (2011) RALA and RALBP1 regulate mitochondrial fission at mitosis. Nat Cell Biol 13(9):1108-1115

52. Kopeikina KJ, Carlson GA, Pitstick R, Ludvigson AE, Peters A, Luebke JI, Koffie RM, Frosch MP, Hyman BT, Spires-Jones TL (2011) Tau accumulation causes mitochondrial distribution deficits in neurons in a mouse model of tauopathy and in human AD brain. Am J Pathol 179(4):2071-2082

53. Lackner LL, Horner JS, Nunnari J (2009) Mechanistic analysis of a dynamin effector. Science 325(5942):874-877

54. Langston JW, Ballard P, Tetrud JW, Irwin I (1983) Chronic Parkinsonism in humans due to a product of meperidine-analog synthesis. Science 219(4587):979-980

55. Lewis MR, Lewis WH (1914) Mitochondria in tissue culture. Science 39(1000):330-333

56. Li Z, Okamoto K, Hayashi Y, Sheng M (2004) The importance of dendritic mitochondria in the morphogenesis and plasticity of spines and synapses. Cell 119(6):873-887

57. Lipinski MM, Zheng B, Lu T, Yan Z, Py BF, Ng A, Xavier RJ, Li C, Yankner BA, Scherzer CR, Yuan J (2010) Genome-wide analysis reveals mechanisms modulating autophagy in normal brain aging and in Alzheimer's disease. Proc Natl Acad Sci USA 107(32):14164-14169

58. Liu X, Hajnoczky G (2009) $\mathrm{Ca}^{+}$-dependent regulation of mitochondrial dynamics by the Miro-Milton complex. Int $\mathbf{J}$ Biochem Cell Biol 41(10):1972-1976

59. Lopes JP, Agostinho P (2011) Cdk5: multitasking between physiological and pathological conditions. Prog Neurobiol 94(1):49-63

60. Manczak M, Calkins MJ, Reddy PH (2011) Impaired mitochondrial dynamics and abnormal interaction of amyloid beta with mitochondrial protein Drp1 in neurons from patients with Alzheimer's disease: implications for neuronal damage. Hum Mol Genet 20(13):2495-2509

61. Mariani E, Polidori MC, Cherubini A, Mecocci P (2005) Oxidative stress in brain aging, neurodegenerative and vascular diseases: an overview. J Chromatogr B Anal Technol Biomed Life Sci 827(1):65-75

62. McNaught KS, Belizaire R, Isacson O, Jenner P, Olanow CW (2003) Altered proteasomal function in sporadic Parkinson's disease. Exp Neurol 179(1):38-46

63. Mears JA, Lackner LL, Fang S, Ingerman E, Nunnari J, Hinshaw JE (2011) Conformational changes in Dnm1 support a contractile mechanism for mitochondrial fission. Nat Struct Mol Biol 18(1):20-26

64. Menn B, Bach S, Blevins TL, Campbell M, Meijer L, Timsit S (2010) Delayed treatment with systemic (S)-roscovitine provides neuroprotection and inhibits in vivo CDK5 activity increase in animal stroke models. PLoS ONE 5(8):e12117

65. Meredith GE, Sonsalla PK, Chesselet MF (2008) Animal models of Parkinson's disease progression. Acta Neuropathol 115(4):385-398

66. Meuer K, Suppanz IE, Lingor P, Planchamp V, Goricke B, Fichtner L, Braus GH, Dietz GP, Jakobs S, Bahr M, Weishaupt JH (2007) Cyclin-dependent kinase 5 is an upstream regulator of mitochondrial fission during neuronal apoptosis. Cell Death Differ 14(4):651-661

67. Mitra K, Wunder C, Roysam B, Lin G, Lippincott-Schwartz J (2009) A hyperfused mitochondrial state achieved at G1-S regulates cyclin $\mathrm{E}$ buildup and entry into $\mathrm{S}$ phase. Proc Natl Acad Sci USA 106(29):11960-11965

68. Montessuit S, Somasekharan SP, Terrones O, Lucken-Ardjomande S, Herzig S, Schwarzenbacher R, Manstein DJ, BossyWetzel E, Basanez G, Meda P, Martinou JC (2010) Membrane remodeling induced by the dynamin-related protein Drp1 stimulates Bax oligomerization. Cell 142(6):889-901 
69. Mopert K, Hajek P, Frank S, Chen C, Kaufmann J, Santel A (2009) Loss of Drp1 function alters OPA1 processing and changes mitochondrial membrane organization. Exp Cell Res 315(13):2165-2180

70. Morris RL, Hollenbeck PJ (1993) The regulation of bidirectional mitochondrial transport is coordinated with axonal outgrowth. J Cell Sci 104(Pt 3):917-927

71. Nakada K, Inoue K, Ono T, Isobe K, Ogura A, Goto YI, Nonaka I, Hayashi JI (2001) Inter-mitochondrial complementation: mitochondria-specific system preventing mice from expression of disease phenotypes by mutant mtDNA. Nat Med 7(8):934-940

72. Nakamura K, Nemani VM, Azarbal F, Skibinski G, Levy JM, Egami K, Munishkina L, Zhang J, Gardner B, Wakabayashi J, Sesaki H, Cheng Y, Finkbeiner S, Nussbaum RL, Masliah E, Edwards RH (2011) Direct membrane association drives mitochondrial fission by the Parkinson disease-associated protein alpha-synuclein. J Biol Chem 286(23):20710-20726

73. Nakamura T, Cieplak P, Cho DH, Godzik A, Lipton SA (2010) S-nitrosylation of Drp1 links excessive mitochondrial fission to neuronal injury in neurodegeneration. Mitochondrion 10(5):573578

74. Napoli I, Neumann H (2009) Microglial clearance function in health and disease. Neuroscience 158(3):1030-1038

75. Narendra D, Tanaka A, Suen DF, Youle RJ (2008) Parkin is recruited selectively to impaired mitochondria and promotes their autophagy. J Cell Biol 183(5):795-803

76. Narendra DP, Jin SM, Tanaka A, Suen DF, Gautier CA, Shen J, Cookson MR, Youle RJ (2010) PINK1 is selectively stabilized on impaired mitochondria to activate Parkin. PLoS Biol 8(1):e1000298

77. Ong SB, Subrayan S, Lim SY, Yellon DM, Davidson SM, Hausenloy DJ (2010) Inhibiting mitochondrial fission protects the heart against ischemia/reperfusion injury. Circulation 121(18):2012-2022

78. Ono T, Isobe K, Nakada K, Hayashi JI (2001) Human cells are protected from mitochondrial dysfunction by complementation of DNA products in fused mitochondria. Nat Genet 28(3):272-275

79. Otera H, Mihara K (2011) Discovery of the membrane receptor for mitochondrial fission GTPase Drp1. Small Gtpases 2(3):167-172

80. Palade GE (1953) An electron microscope study of the mitochondrial structure. J Histochem Cytochem 1(4):188-211

81. Park KS, Wiederkehr A, Kirkpatrick C, Mattenberger Y, Martinou JC, Marchetti P, Demaurex N, Wollheim CB (2008) Selective actions of mitochondrial fission/fusion genes on metabolism-secretion coupling in insulin-releasing cells. J Biol Chem 283(48):33347-33356

82. Park SW, Kim KY, Lindsey JD, Dai Y, Heo H, Nguyen DH, Ellisman MH, Weinreb RN, Ju WK (2011) A selective inhibitor of drp1, mdivi-1, increases retinal ganglion cell survival in acute ischemic mouse retina. Invest Ophthalmol Vis Sci 52(5):2837-2843

83. Park YY, Lee S, Karbowski M, Neutzner A, Youle RJ, Cho H (2010) Loss of MARCH5 mitochondrial E3 ubiquitin ligase induces cellular senescence through dynamin-related protein 1 and mitofusin 1. J Cell Sci 123(Pt 4):619-626

84. Parone PA, James DI, Da Cruz S, Mattenberger Y, Donze O, Barja F, Martinou JC (2006) Inhibiting the mitochondrial fission machinery does not prevent Bax/Bak-dependent apoptosis. Mol Cell Biol 26(20):7397-7408

85. Prass K, Royl G, Lindauer U, Freyer D, Megow D, Dirnagl U, Stockler-Ipsiroglu G, Wallimann T, Priller J (2007) Improved reperfusion and neuroprotection by creatine in a mouse model of stroke. J Cereb Blood Flow Metab 27(3):452-459
86. Qi X, Disatnik MH, Shen N, Sobel RA, Mochly-Rosen D (2011) Aberrant mitochondrial fission in neurons induced by protein kinase $\mathrm{C}\{$ delta $\}$ under oxidative stress conditions in vivo. Mol Biol Cell 22(2):256-265

87. Rintoul GL, Filiano AJ, Brocard JB, Kress GJ, Reynolds IJ (2003) Glutamate decreases mitochondrial size and movement in primary forebrain neurons. J Neurosci 23(21):7881-7888

88. Rosenstock TR, de Brito OM, Lombardi V, Louros S, Ribeiro M, Almeida S, Ferreira IL, Oliveira CR, Rego AC (2011) FK506 ameliorates cell death features in Huntington's disease striatal cell models. Neurochem Int 59(5):600-609

89. Rowland KC, Irby NK, Spirou GA (2000) Specialized synapseassociated structures within the calyx of Held. J Neurosci 20(24):9135-9144

90. Sandebring A, Thomas KJ, Beilina A, van der Brug M, Cleland MM, Ahmad R, Miller DW, Zambrano I, Cowburn RF, Behbahani H, Cedazo-Minguez A, Cookson MR (2009) Mitochondrial alterations in PINK1 deficient cells are influenced by calcineurin-dependent dephosphorylation of dynamin-related protein 1. PLoS One 4(5):e5701

91. Schapira AH, Cooper JM, Dexter D, Jenner P, Clark JB, Marsden CD (1989) Mitochondrial complex I deficiency in Parkinson's disease. Lancet 1(8649):1269

92. Schon EA, Przedborski S (2011) Mitochondria: the next (neurode)generation. Neuron 70(6):1033-1053

93. Scorrano L, Ashiya M, Buttle K, Weiler S, Oakes SA, Mannella CA, Korsmeyer SJ (2002) A distinct pathway remodels mitochondrial cristae and mobilizes cytochrome c during apoptosis. Dev Cell 2(1):55-67

94. Sheridan C, Martin SJ (2010) Mitochondrial fission/fusion dynamics and apoptosis. Mitochondrion 10(6):640-648

95. Shirendeb U, Reddy AP, Manczak M, Calkins MJ, Mao P, Tagle DA, Reddy PH (2011) Abnormal mitochondrial dynamics, mitochondrial loss and mutant huntingtin oligomers in Huntington's disease: implications for selective neuronal damage. Hum Mol Genet 20(7):1438-1455

96. Smirnova E, Shurland DL, Ryazantsev SN, van der Bliek AM (1998) A human dynamin-related protein controls the distribution of mitochondria. J Cell Biol 143(2):351-358

97. Song W, Chen J, Petrilli A, Liot G, Klinglmayr E, Zhou Y, Poquiz P, Tjong J, Pouladi MA, Hayden MR, Masliah E, Ellisman M, Rouiller I, Schwarzenbacher R, Bossy B, Perkins G, Bossy-Wetzel E (2011) Mutant huntingtin binds the mitochondrial fission GTPase dynamin-related protein-1 and increases its enzymatic activity. Nat Med 17(3):377-382

98. Spillantini MG, Schmidt ML, Lee VM, Trojanowski JQ, Jakes R, Goedert M (1997) Alpha-synuclein in Lewy bodies. Nature 388(6645):839-840

99. Strauss M, Hofhaus G, Schroder RR, Kuhlbrandt W (2008) Dimer ribbons of ATP synthase shape the inner mitochondrial membrane. EMBO J 27(7):1154-1160

100. Taguchi N, Ishihara N, Jofuku A, Oka T, Mihara K (2007) Mitotic phosphorylation of dynamin-related GTPase Drp1 participates in mitochondrial fission. J Biol Chem 282(15):1152111529

101. Tian C, Murrin LC, Zheng JC (2009) Mitochondrial fragmentation is involved in methamphetamine-induced cell death in rat hippocampal neural progenitor cells. PLoS ONE 4(5):e5546

102. Tondera D, Grandemange S, Jourdain A, Karbowski M, Mattenberger Y, Herzig S, Da Cruz S, Clerc P, Raschke I, Merkwirth C, Ehses S, Krause F, Chan DC, Alexander C, Bauer C, Youle R, Langer T, Martinou JC (2009) SLP-2 is required for stress-induced mitochondrial hyperfusion. EMBO J 28(11): $1589-1600$

103. Twig G, Elorza A, Molina AJ, Mohamed H, Wikstrom JD, Walzer G, Stiles L, Haigh SE, Katz S, Las G, Alroy J, Wu M, Py 
BF, Yuan J, Deeney JT, Corkey BE, Shirihai OS (2008) Fission and selective fusion govern mitochondrial segregation and elimination by autophagy. EMBO J 27(2):433-446

104. Uo T, Dworzak J, Kinoshita C, Inman DM, Kinoshita Y, Horner PJ, Morrison RS (2009) Drp1 levels constitutively regulate mitochondrial dynamics and cell survival in cortical neurons. Exp Neurol 218(2):274-285

105. Varadi A, Johnson-Cadwell LI, Cirulli V, Yoon Y, Allan VJ, Rutter GA (2004) Cytoplasmic dynein regulates the subcellular distribution of mitochondria by controlling the recruitment of the fission factor dynamin-related protein-1. J Cell Sci $117(\mathrm{Pt}$ 19):4389-4400

106. Verstreken P, Ly CV, Venken KJ, Koh TW, Zhou Y, Bellen HJ (2005) Synaptic mitochondria are critical for mobilization of reserve pool vesicles at Drosophila neuromuscular junctions. Neuron 47(3):365-378

107. von Campenhausen S, Bornschein B, Wick R, Botzel K, Sampaio C, Poewe W, Oertel W, Siebert U, Berger K, Dodel R (2005) Prevalence and incidence of Parkinson's disease in Europe. Eur Neuropsychopharmacol 15(4):473-490

108. Wakabayashi J, Zhang Z, Wakabayashi N, Tamura Y, Fukaya M, Kensler TW, Iijima M, Sesaki H (2009) The dynamin-related GTPase Drp1 is required for embryonic and brain development in mice. J Cell Biol 186(6):805-816

109. Walker FO (2007) Huntington's disease. Lancet 369(9557):218228

110. Walsh B, Tonkonogi M, Soderlund K, Hultman E, Saks V, Sahlin K (2001) The role of phosphorylcreatine and creatine in the regulation of mitochondrial respiration in human skeletal muscle. J Physiol 537(Pt 3):971-978

111. Wang H, Song P, Du L, Tian W, Yue W, Liu M, Li D, Wang B, Zhu Y, Cao C, Zhou J, Chen Q (2011) Parkin ubiquitinates Drp1 for proteasome-dependent degradation: implication of dysregulated mitochondrial dynamics in Parkinson disease. J Biol Chem 286(13):11649-11658

112. Wang JX, Jiao JQ, Li Q, Long B, Wang K, Liu JP, Li YR, Li PF (2011) miR-499 regulates mitochondrial dynamics by targeting calcineurin and dynamin-related protein-1. Nat Med 17(1):71-78

113. Wang X, Su B, Lee HG, Li X, Perry G, Smith MA, Zhu X (2009) Impaired balance of mitochondrial fission and fusion in Alzheimer's disease. J Neurosci 29(28):9090-9103

114. Wang X, Su B, Siedlak SL, Moreira PI, Fujioka H, Wang Y, Casadesus G, Zhu X (2008) Amyloid-beta overproduction causes abnormal mitochondrial dynamics via differential modulation of mitochondrial fission/fusion proteins. Proc Natl Acad Sci USA 105(49): 19318-19323

115. Wasiak S, Zunino R, McBride HM (2007) Bax/Bak promote sumoylation of DRP1 and its stable association with mitochondria during apoptotic cell death. J Cell Biol 177(3):439-450

116. Wasilewski M, Scorrano L (2009) The changing shape of mitochondrial apoptosis. Trends Endocrinol Metab 20(6):287294

117. Waterham HR, Koster J, van Roermund CW, Mooyer PA, Wanders RJ, Leonard JV (2007) A lethal defect of mitochondrial and peroxisomal fission. N Engl J Med 356(17):1736-1741

118. Yoon Y, Pitts KR, Dahan S, McNiven MA (1998) A novel dynamin-like protein associates with cytoplasmic vesicles and tubules of the endoplasmic reticulum in mammalian cells. J Cell Biol 140(4):779-793

119. Young KW, Pinon LG, Bampton ET, Nicotera P (2010) Different pathways lead to mitochondrial fragmentation during apoptotic and excitotoxic cell death in primary neurons. J Biochem Mol Toxicol 24(5):335-341

120. Yuan H, Gerencser AA, Liot G, Lipton SA, Ellisman M, Perkins GA, Bossy-Wetzel E (2007) Mitochondrial fission is an upstream and required event for bax foci formation in response to nitric oxide in cortical neurons. Cell Death Differ 14(3):462-471

121. Zhao J, Liu T, Jin S, Wang X, Qu M, Uhlen P, Tomilin N, Shupliakov O, Lendahl U, Nister M (2011) Human MIEF1 recruits Drp1 to mitochondrial outer membranes and promotes mitochondrial fusion rather than fission. EMBO J 30(14):27622778

122. Zuchner S, Mersiyanova IV, Muglia M, Bissar-Tadmouri N, Rochelle J, Dadali EL, Zappia M, Nelis E, Patitucci A, Senderek J, Parman Y, Evgrafov O, Jonghe PD, Takahashi Y, Tsuji S, Pericak-Vance MA, Quattrone A, Battaloglu E, Polyakov AV, Timmerman V, Schroder JM, Vance JM (2004) Mutations in the mitochondrial GTPase mitofusin 2 cause Charcot-Marie-Tooth neuropathy type 2A. Nat Genet 36(5):449-451

123. Zunino R, Braschi E, Xu L, McBride HM (2009) Translocation of SenP5 from the nucleoli to the mitochondria modulates DRP1-dependent fission during mitosis. J Biol Chem 284(26): 17783-17795

124. Zunino R, Schauss A, Rippstein P, Andrade-Navarro M, McBride HM (2007) The SUMO protease SENP5 is required to maintain mitochondrial morphology and function. J Cell Sci 120(Pt 7):1178-1188 